\title{
Bee venom phospholipase A2 prevents prion peptide induced-cell death in neuronal cells
}

\author{
JAE-KYO JEONG, MYUNG-HEE MOON, BUM-CHUL BAE, YOU-JIN LEE, \\ JAE-WON SEOL and SANG-YOUEL PARK \\ Center for Healthcare Technology Development, Korea Zoonoses Research Institute, College of \\ Veterinary Medicine, Chonbuk National University, Jeonju 561-756, Republic of Korea
}

Received May 11, 2011; Accepted June 14, 2011

DOI: $10.3892 /$ ijmm.2011.730

\begin{abstract}
Bee venom phospholipase A2 (bvPLA2) is a prototypic group III enzyme which consists of unique N-terminal and C-terminal domains and a central secretory PLA2 (sPLA2) domain. This SPLA2 domain is highly homologous with human group III sPLA2. Current evidence suggests that group III sPLA2 may affect some neuronal functions, such as neuritogenesis, neurotransmitter release and neuronal survival. The prion diseases are neurodegenerative disorders characterized by the conversion of the normal cellular prion (PrPC) to the misfolded isoform scrapie prion protein (PrPSc). PrPSc accumulation in the central nervous system (CNS) leads to neurotoxicity by inhibition of the PI3K/AKT pathway or activation of $\mathrm{p} 38$ mitogen-activated protein kinase (MAPK) pathways. In the present study, we found that bvPLA2 inhibited prion protein (PrP) fragment (106-126)-induced neuronal cell death. $\operatorname{PrP}(106-$ 126)-mediated increase of p-p38 MAPK and cleaved caspases and decrease of p-AKT were blocked by bvPLA2 treatment. These results indicate that increasing PLA2, including the group III sPLA2 is key to regulating PrP(106-126)-mediated neurotoxicity. Taken together, the results of this study suggest that specific modulation of PLA2 appears to prevent neuronal cell death caused by prion peptides.
\end{abstract}

\section{Introduction}

Transmissible spongiform encephalopathies, also known as prion diseases, are a family of progressive neurodegenerative disorders characterized by central nervous system (CNS) spongiosis, neuronal loss, and accumulation of scrapie prion protein (PrPSc) in the brain (1). One of the main characteristics of prion disease pathogenesis is the formation of PrPSc, a

Correspondence to: Dr Sang-Youel Park, College of Veterinary Medicine, Chonbuk National University, Jeonju, Jeonbuk 561-756, Republic of Korea

E-mail: sypark@chonbuk.ac.kr

Key words: bee venom phospholipase A2, prion protein (106-126), prion disease, secretory phospholipase A2 proteinase K-resistant prion protein ( $\mathrm{PrP})$ which is a misfolded form of normal cellular prion protein (PrPC) (2). PrPSc is derived from PrPC as a result of conformational conversion $(1,3,4)$. The presence of PrPC, thus, is required for PrPSc accumulation within the CNS and the progression of prion diseases $(5,6)$. The membrane-linked form of PrPC, which is anchored to the cell surface via a glycosyl-phosphatidylinositol (GPI) linkage, is expressed in neuronal cells $(7,8)$. PrPC has various physical properties, including protection from oxidative stress, transmembrane signaling, control of copper homeostasis, and cell adhesion $(9,10)$.

Although the mechanisms of PrPSc neurotoxicity are yet unclear, recent studies support the hypothesis that PrPSc induces neuronal cell death and activates caspase-3 through the p38-JNK mitogen-activated protein kinase (MAPK) pathway $(2,11,12)$. Seo et al studying PrP peptide 106-126 [PrP(106-126)]-treated SH-SY5Y cells, have shown that $\operatorname{PrP}(106-126)$-induced neurotoxicity is decreased by repression of p38 MAPK activation with exposure to the SIRT1 activator, resveratrol (13).

$\operatorname{PrP}(106-126)$ contains the amino acid residues 106-126 of PrPC and possesses many characteristics of the entire PrPSc including the capacity to cause neurotoxicity in hippocampal neuronal cells which catalyze the aggregation of endogenous PrPC to fibril accumulation $(14,15)$. These properties are useful for the in vitro study of PrPSc pathogenesis (16).

Phospholipase A2 (PLA2) is a lipolytic enzyme that catalyzes the hydrolysis of the sn-2 ester linkage of glycerophospholipids to liberate free fatty acids and lysophospholipids (17). Cellular functions of PLA2s are known to modulate the release of arachidonic acid and the generation of eicosanoids, which are potent inflammatory mediators (18). Furthermore, PLA2 plays a central role in host defense, differentiation (19), and membrane remodeling (20). PLA2 enzymes are found in a variety of sources including the mammalian pancreas, synovial fluids, and the venom of organisms including reptiles, insects, amphibians, arachnids, and coelenterates (21). The PLA2 superfamily is subdivided into 15 groups on the basis of the homology of their structure, source, and localization (22). Distribution among these groups can be classified into secretory PLA2 (sPLA2, groups I, II, III, V, IX, X, XI, XII, XIII and XIV), $\mathrm{Ca}^{2+}$-dependent cytosolic PLA2 (cPLA2, group IV), and $\mathrm{Ca}^{2+}$-independent cytosolic PLA2 (iPLA2, group VI) (20). 
The sPLA2 family represents a group of structurally homologous, $\mathrm{Ca}^{2+}$-dependent, low-molecular weight enzymes. Individual sPLA2s exhibit specific tissue and cellular localizations. As such, it is proposed that they perform distinct biological roles by activating different target substrates (23). Group IA sPLA2 is involved in digestion, while, group II sPLA2 is involved in antibacterial defense (22). Additionally, the function of group III sPLA2 is as a regulator of inflammation, proliferation, fertility, and maturation in mammals $(19,24,25)$.

Group III sPLA2 is a typical sPLA2 member, homologous with bee venom phospholipase A2 (bvPLA2) compared to other mammalian sPLA2s (26). Recent studies suggested that Group III sPLA2 mediates atherosclerosis and differentiation of sperm maturation and fertility in transgenic mice $(24,25)$. Additionally, this enzyme is known to regulate neuronal outgrowth and survival by activation of AKT (19). One report suggested its use as a pharmacological therapy for Alzheimer's disease via enhanced $\alpha$-secretase-dependent amyloid precursor protein (APP) processing by regulation of membrane fluidity (27); however, the effect of group III sPLA2 enzymes on $\operatorname{PrP}(106-126)$-mediated neurotoxicity has not been reported.

Thus, the present study focused on the influence of byPLA2 on the regulation of $\operatorname{PrP}(106-126)$-induced apoptosis in neuronal cells. The results showed that neuronal cells exposed to bvPLA2 experienced decreased $\operatorname{PrP}(106-126)$-mediated neurotoxicity. The present results suggest that regulation of group III PLA2 is a viable therapeutic strategy for neurodegenerative diseases including prion disease.

\section{Materials and methods}

Cell culture. Human neuroblastoma cell lines (SH-SY5Y) were obtained from the American Type Culture collection (ATCC, Rockville, MD, USA). Cells were cultured in Minimum Essential Medium (Hyclone Laboratories, Logan, UT, USA) that contained $10 \%$ fetal bovine serum (InvitrogenGibco, Grand Island, NY, USA) and gentamycin $(0.1 \mathrm{mg} / \mathrm{ml})$ in a humidified incubator maintained at $37^{\circ} \mathrm{C}$ and $5 \% \mathrm{CO}_{2}$.

$\operatorname{PrP}(106-126)$ treatment. Synthetic $\operatorname{PrP}(106-126)$ (sequence, Lys-Thr-Asn-Met-Lys-His-Met-Ala-Gly-Ala-Ala-Ala-Ala-GlyAla-Val-Val-Gly-Gly-Leu-Gly) peptides were synthesized from Peptron (Seoul, Korea). The peptides were dissolved in sterile DMSO at a concentration of $12.5 \mathrm{mM}$ and stored at $-80^{\circ} \mathrm{C}$.

Lactate dehydrogenase (LDH) assay. Cytotoxicity was assessed by the LDH assay in the supernatant medium using a LDH Cytotoxicity Detection kit (Takara Bio, Inc., Tokyo, Japan) according to the manufacturer's protocol. The LDH activity was determined by measuring the absorbance at $490 \mathrm{~nm}$ using a microplate reader (Spectra Max M2, Molecular Devices, USA).

Annexin $V$ assay. Apoptosis was assessed by the Annexin $\mathrm{V}$ assay in the detached cells using an Annexin V assay kit (Santa Cruz Biotechnology) according to the manufacturer's protocol. Annexin $\mathrm{V}$ measurement was determined by measuring the fluorescence at excitation $488 \mathrm{~nm}$ and emission 525/30 using a Guava easyCyte HT (Millipore).
Terminal deoxynucleotidyl transferase dUTP nick end labeling (TUNEL) assay. TUNEL analysis was performed to measure the degree of cellular apoptosis using an in situ ApoBrdU DNA fragmentation assay kit (BioVision, San Francisco, CA, USA) following the manufacturer's instructions. Cells were washed with phosphate buffer saline (PBS) and fixed with paraformaldehyde for $15 \mathrm{~min}$. Cells were pre-incubated with $50 \mu \mathrm{l}$ DNA-labelling solution (10 $\mu \mathrm{l}$ TdT reaction buffer, $0.75 \mu \mathrm{l}$ TdT enzyme, $8 \mu \mathrm{l} \mathrm{Br}$-dUTP) for $1 \mathrm{~h}$ at $37^{\circ} \mathrm{C}$ and then cells were incubated with $5 \mu 1$ anti-BrdU-FITC antibody for $0.5 \mathrm{~h}$ at room temperature $\left(20^{\circ} \mathrm{C}\right)$. Finally, cells were mounted with DakoCytomation fluorescent medium and visualized usiong fluorescence microscopy. Cells were counterstained with propodium iodide (PI) to show all cell nuclei.

Western blot analyses. SH-SY5Y was lysed in a lysis buffer (25 mM HEPES; pH 7.4, $100 \mathrm{mM} \mathrm{NaCl}, 1 \mathrm{mM}$ EDTA, $5 \mathrm{mM}$ $\mathrm{MgCl}_{2}, 0.1 \mathrm{mM}$ DTT and protease inhibitor mixture). Proteins were electrophoretically resolved on a $10-15 \%$ sodium dodecyl sulfate (SDS) gel, and immunoblotting was performed as previously described. Equal amounts of lysate protein were resolved on a $10-15 \%$ SDS-polyacrylamide gel and electrophoretically transferred to a nitrocellulose membrane. Immunoreactivity was detected through sequential incubation with horseradish peroxidase-conjugated secondary antibodies and ECL reagents. The antibodies used for immunoblotting were caspase-3, p38, phospho-p38 (all from Cell Signaling Technology), AKT (Calbiochem), phospho-AKT (Calbiochem) and $\beta$-actin (Sigma). Images were examined using an Fusion-FX7 imaging system (Vilber Lourmat).

\section{Results}

$\operatorname{PrP}(106-126)$-mediated neurotoxicity is attenuated by bvPLA2 treatment in SH-SY5Y neuroblastoma cells. The influence of bvPLA2 on $\operatorname{PrP}(106-126)$-mediated neurotoxicity in SH-SY5Y cells was determined by the Annexin V assay. SH-SY5Y cells were exposed to bvPLA2 with or without $\operatorname{PrP}(106-126)$. Treatment of SH-SY5Y cells with $\operatorname{PrP}(106-$ 126), but not bvPLA2, resulted in neurotoxicity, identified by the fact that Annexin $\mathrm{V}$ positive cells were $59.51 \%$ increased after $24 \mathrm{~h}$ (Fig. 1A and B). However, treatment with bvPLA2 inhibited PrP(106-126)-mediated neurotoxicity in SH-SY5Y cells (Fig. 1). These results were confirmed by examination of LDH-releasing levels (Fig. 1D). Consistent with these results, the TUNEL assay and immunoblotting of activated caspase-3 also showed that bvPLA2 treatment completely inhibited $\operatorname{PrP}(106-126)$-induced apoptosis (Figs. 1C and 2). These result suggest that bvPLA2 attenuates $\operatorname{PrP}(106-126)$-induced apoptosis in neuronal cells.

Administration of bvPLA2 inhibits $\operatorname{PrP}(106-126)$-induced neuronal cell death by regulating the activation of $A K T$ and p38 MAPK proteins. Because group III sPLA2 can regulate both neuronal survival and neurite outgrowth via regulation of AKT (19), we investigated whether bvPLA2 had an effect on AKT, p38 MAPK and caspase-3 protein levels. SH-SY5Y cells were pre-incubated with different concentrations of bvPLA2 for $1 \mathrm{~h}$ then exposed to $\operatorname{PrP}(106-126)$ (Fig. 2). The $\operatorname{PrP}(106-126)$-treated cells showed increased phosphorylated- 
A

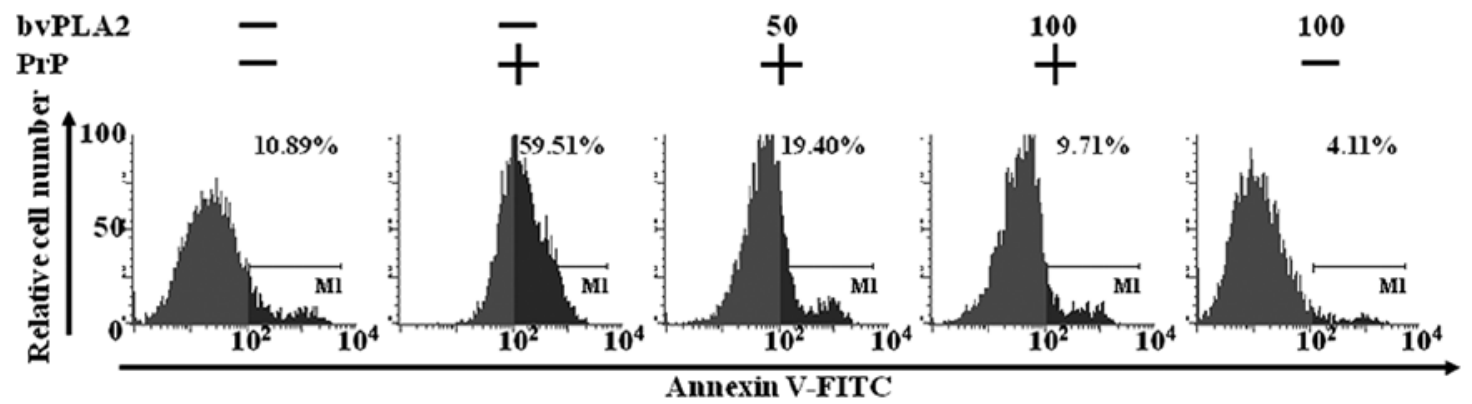

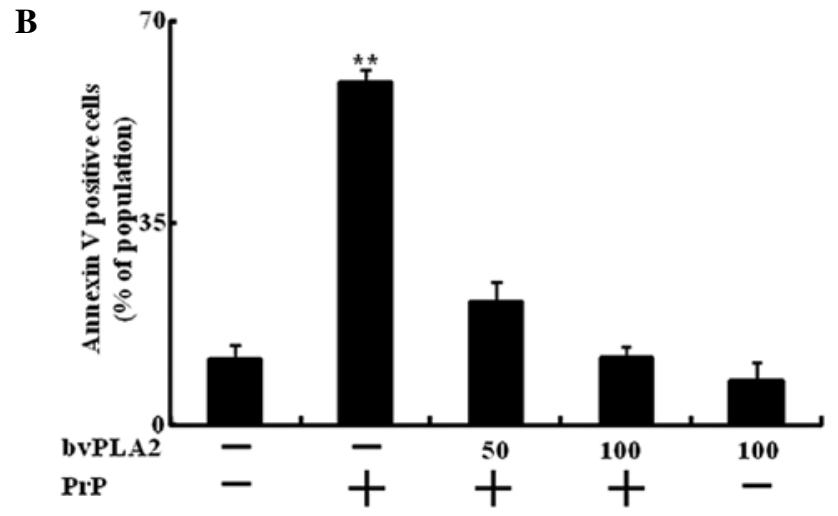

D

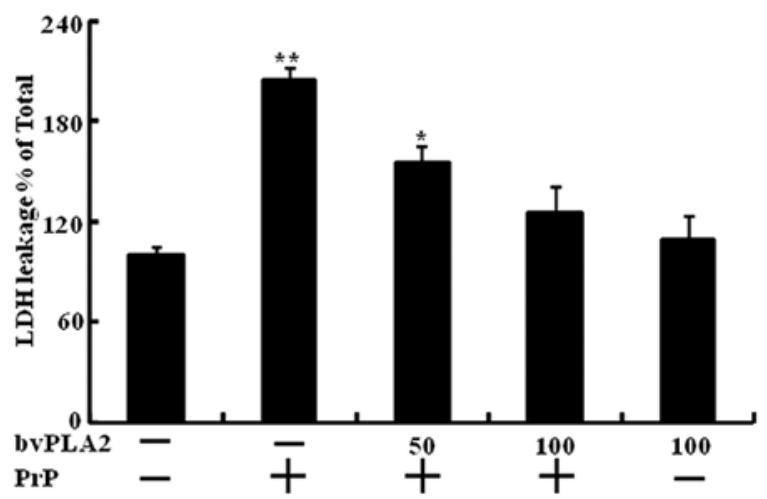

p38 protein levels and decreased phosphorylated-AKT protein levels. By contrast, bvPLA2 administration was associated with decreased p-p38 protein levels and increased p-AKT protein levels in SH-SY5Y cells treated with PrP(106-126) (Fig. 2), in a dose dependent manner. The results indicate that treatment with bvPLA2 prevents prion peptide-induced apoptosis by regulating AKT and p38 MAPK activation.

To determine whether bvPLA2 operates by activating AKT and p38 MAPK proteins to inhibit PrP(106-126)-induced apoptosis, SH-SY5Y cells were pre-treated with bvPLA2, AKT inhibitor, wortmannin, and p38 inhibitor, SB-203580, and then exposed to $\operatorname{PrP}(106-126)$ (Figs. 3 and 4). First, we evaluated the influence of AKT activation on the protective effect of bvPLA2 on cells after exposure to $\operatorname{PrP}(106-126)$. Administration of bvPLA2 decreased Annexin $\mathrm{V}$ positive cells following treatment with $\operatorname{PrP}(106-126)$, but this effect was blocked by treatment with the AKT inhibitor wortmannin (Fig. 3A and B). These results were confirmed by examination of LDH-releasing levels (Fig. 3D). Consistent with these findings, the TUNEL assay and Western blot analysis also showed that pre-treatment with wortmannin blocked the protective
C

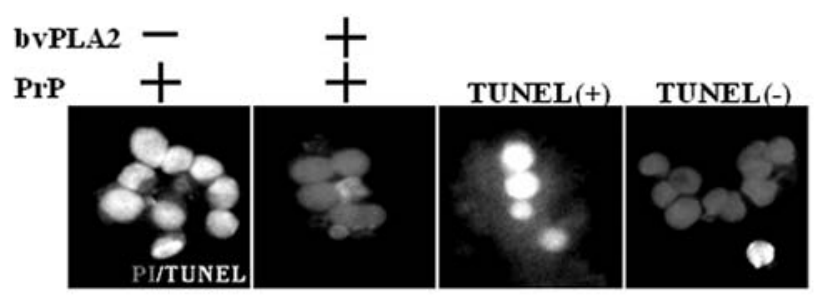

Figure 1. Bee venom PLA2 (bvPLA2) protects neuronal cells from $\operatorname{PrP}(106-$ 126)-induced neuronal cell death. (A) SH-SY5Y cells were pretreated with bvPLA2 $(1 \mathrm{~h})$ in a dose-dependent manner, and then exposed to $50 \mu \mathrm{M}$ $\operatorname{PrP}(106-126)$ for $23 \mathrm{~h}$. Cell viability was measured by the Annexin V assay. Cells were treated with FITC-Annexin V, which binds to phosphatidylserine on the plasma membrane during apoptosis. M1 represents the population of Annexin V positive cells. (B) The bar graph indicates the means of the Annexin V positive cells. (C) Representative immunofluorescence images of TUNEL-positive (white) SH-SY5Y cells at $1 \mathrm{~h}$ after exposure to $50 \mu \mathrm{M}$ of $\operatorname{PrP}(106-126)$ in the absence or presence of bvPLA2. The cells were counterstained with PI (gray) to show all cell nuclei. (D) Cells were treated with the indicated concentrations of $\operatorname{PrP}(106-126)$ for $23 \mathrm{~h}$ after exposure to the bvPLA 2 for $1 \mathrm{~h}$, and release of lactate dehydrogenase into the cell culture supernatant from damaged cells was measured. Magnification, $x 400$; scale bar, $10 \mu \mathrm{m}$. The bar graph indicates the mean $\pm \mathrm{SEM}(\mathrm{n}=3) .{ }^{*} \mathrm{P}<0.05,{ }^{* *} \mathrm{P}<0.01$, significant differences between control and each treatment group.

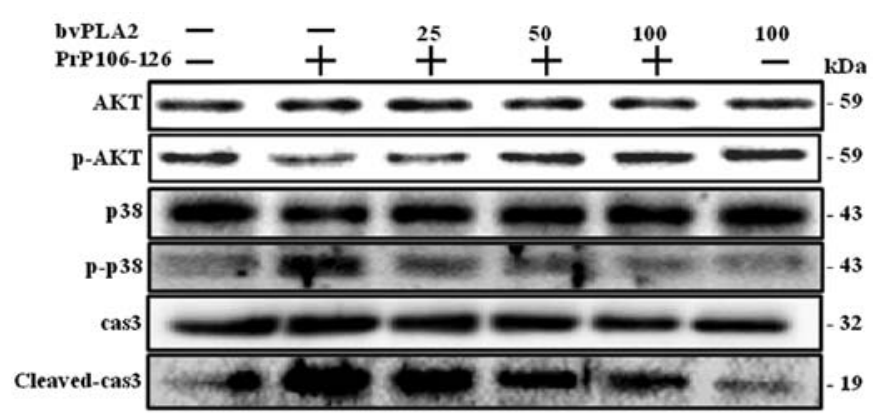

Figure 2. Effects of bee venom PLA2 (bvPLA2) treatment on AKT and p38 phosphorylations on PrP(106-126)-mediated neuronal cell death. SH-SY5Y cells were pretreated with bvPLA2 $(1 \mathrm{~h})$ in a dose-dependent manner, and then exposed to $50 \mu \mathrm{M} \operatorname{PrP}(106-126)$ for $15 \mathrm{~h}$. The treated cells were assessed for AKT, p-AKT, p38, p-p38 and caspase-3 and cleaved caspase-3 production by Western blot analysis.

effect of bvPLA2 on $\operatorname{PrP}(106-126)$ neurotoxicity and decreased the activation of AKT (Fig. 3C and E). Next, to determine whether bvPLA2 had a neuroprotective effect associated with 
A
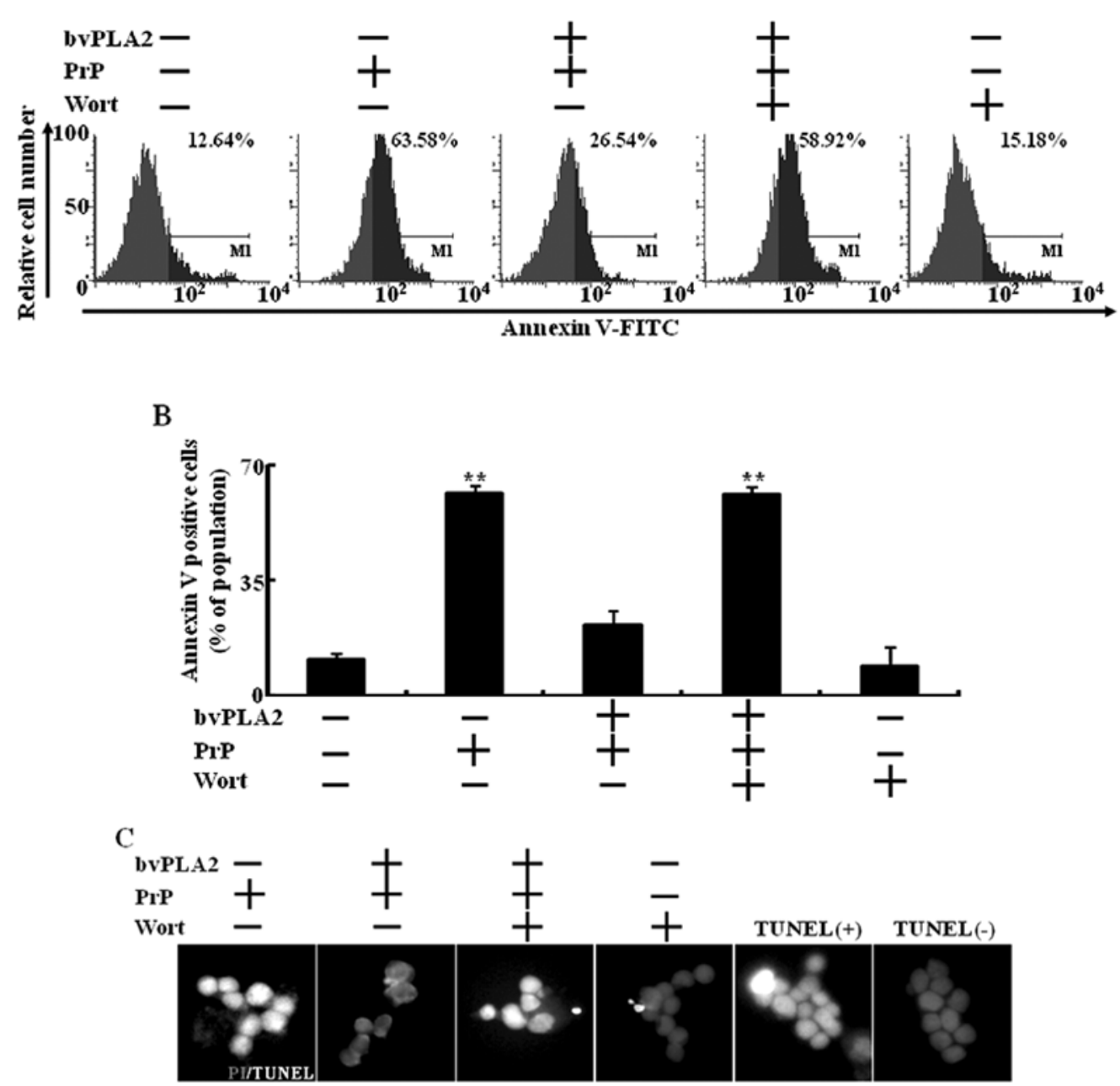

D

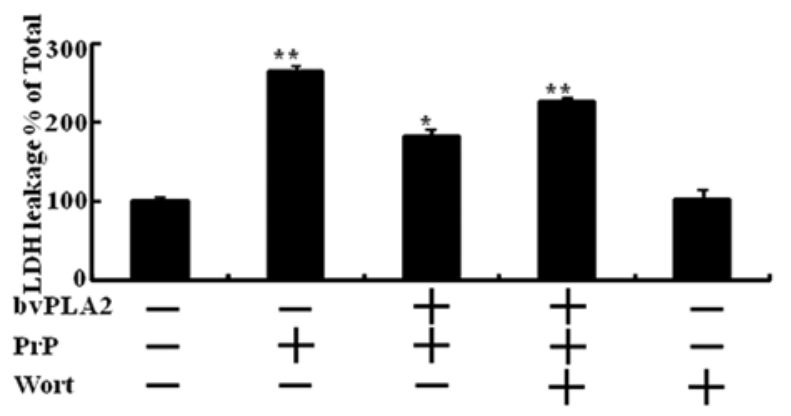

E

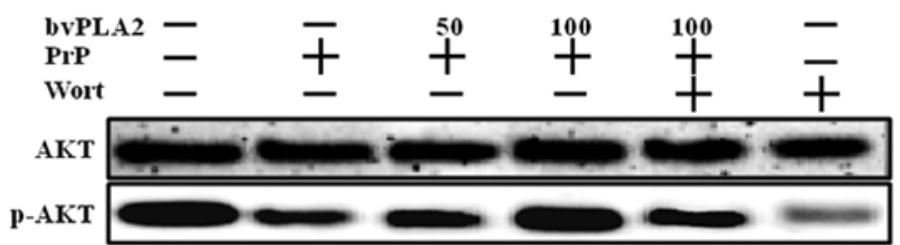

Figure 3. Bee venom PLA2 (bvPLA2) decreases PrP(106-126)-induced neurotoxicity by regulation of AKT signaling in neuronal cells. (A) SH-SY5Y cells were pretreated with $50 \mathrm{nM}$ of bvPLA2 $(1 \mathrm{~h})$ and/or $100 \mathrm{nM}$ of wortmannin (Wort) for $1 \mathrm{~h}$, and then exposed to $50 \mu \mathrm{M} \operatorname{PrP}(106-126)$ for $23 \mathrm{~h}$. Cell viability was measured by the Annexin V assay. M1 represents the population of Annexin V positive cells. (B) The bar graph indicates the means of the Annexin V positive cells. (C) Representative immunofluorescence images of TUNEL-positive (white) SH-SY5Y cells at $1 \mathrm{~h}$ after exposure to 50 $\mu \mathrm{M}$ of PrP(106-126) in the absence or presence of bvPLA2 $(50 \mu \mathrm{M})$ with or without wortmannin $(100 \mathrm{nM})$. The cells were counterstained with PI (gray) to show all cell nuclei. (D) Cells were treated with the indicated concentrations of $\operatorname{PrP}(106-126)$ for $23 \mathrm{~h}$ after exposure to bvPLA2 with or without wortmannin for $1 \mathrm{~h}$, and release of lactate dehydrogenase into the cell culture supernatant from damaged cells was measured. (E) Cells were pretreated with bvPLA2 (1 h) in a dose-dependent manner, and then exposed to $50 \mu \mathrm{M} \operatorname{PrP}(106-126)$ for $15 \mathrm{~h}$ in the absence or presence of $100 \mathrm{nM}$ wortmannin for $1 \mathrm{~h}$. The treated cells were assessed for AKT and p-AKT production by Western blot analysis. Magnification, $\mathrm{x} 400$; scale bar, $10 \mu \mathrm{m}$. The bar graph indicates the mean $\pm \mathrm{SEM}(\mathrm{n}=3)$. ${ }^{*} \mathrm{P}<0.05$, ** $\mathrm{P}<0.01$, significant differences between control and each treatment group. 

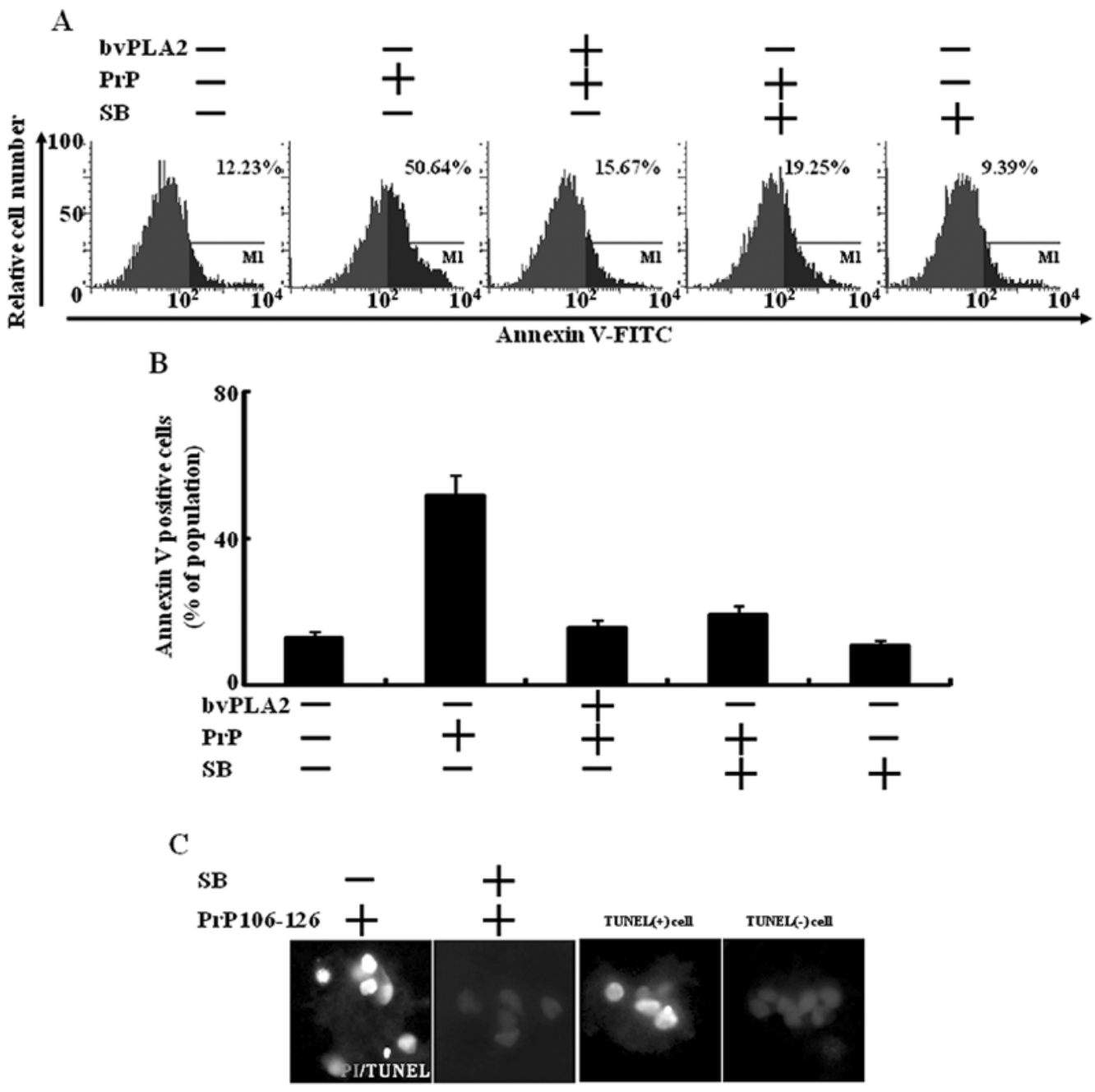

D

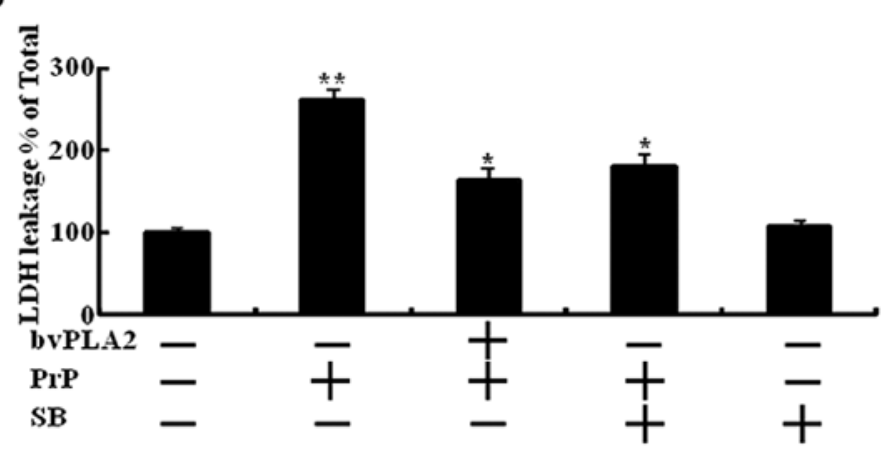

E

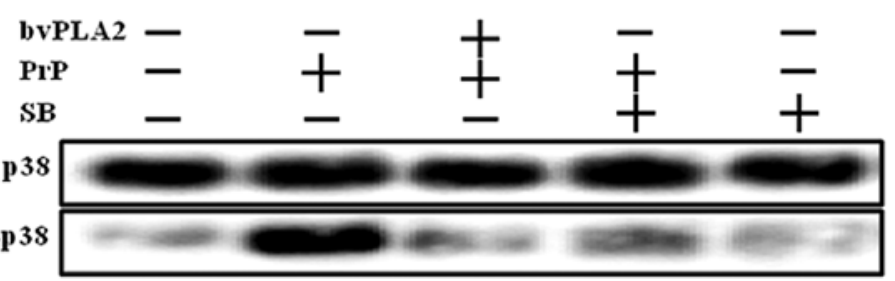

Figure 4. Bee venom PLA2 (bvPLA2) decreases PrP(106-126)-induced neurotoxicity by regulation of p38 signaling in neuronal cells. (A) SH-SY5Y cells were pretreated with $50 \mathrm{nM}$ of bvPLA2 $(1 \mathrm{~h})$ or $100 \mathrm{nM}$ of SB-20358 $(1 \mathrm{~h})$, and then exposed to $50 \mu \mathrm{M} \operatorname{PrP}(106-126)$ for $23 \mathrm{~h}$. Cell viability was measured by the Annexin V assay. M1 represents the population of Annexin V positive cells. (B) The bar graph indicates the means of the Annexin V positive cells. (C) Representative immunofluorescence images of TUNEL-positive (white) SH-SY5Y cells at $1 \mathrm{~h}$ after exposure to $50 \mu \mathrm{M}$ of $\operatorname{PrP}(106-126)$ in the absence or presence of SB-203580. The cells were counterstained with PI (gray) to show all cell nuclei. (D) Cells were treated with the indicated concentrations of $\operatorname{PrP}(106-126)$ for $23 \mathrm{~h}$ after exposure to the bvPLA2 or SB-203580 for $1 \mathrm{~h}$, and release of lactate dehydrogenase into the cell culture supernatant from damaged cells was measured. The bar graph indicates the mean \pm SEM $(n=3)$. (E) SH-SY5Y cells were pretreated with bvPLA2 (1 h) in a dose-dependent manner or exposure to $100 \mathrm{nM}$ of SB-203580 (1 h), and then exposed to $50 \mu \mathrm{M} \operatorname{PrP}(106-126)$ for $15 \mathrm{~h}$. The treated cells were assessed for p38 and p-p38 production by Western blot analysis. Magnification, $\mathrm{x} 400$; scale bar, $10 \mu \mathrm{m}$. Bar graph indicates the mean $\pm \mathrm{SEM}(\mathrm{n}=3)$. ${ }^{*} \mathrm{P}<0.05,{ }^{* * *} \mathrm{P}<0.01$, significant differences between control and each treatment group. 
p38 MAPK inactivation in $\operatorname{PrP}(106-126)$-induced apoptosis, SH-SY5Y cells were pre-treated with bvPLA2 or SB-203580 and then exposed to $\operatorname{PrP}(106-126)$. PrP(106-126)-treated cells demonstrated increased Annexin $\mathrm{V}$ binding to membranes, while bvPLA2 and SB-203580 had no effect on Annexin V binding to membranes. However, bvPLA2 and SB-203580 treatment decreased PrP(106-126)-mediated Annexin V binding to membranes and blocked PrP(106-126)-induced apoptosis (Fig. 4A and B). These results were confirmed by examination of LDH-releasing levels (Fig. 4D). Consistent with these results, the TUNEL assay and Western blot analysis also showed that pre-treatment with bvPLA2 or SB-203580 blocked the neurotoxicity associated with $\operatorname{PrP}(106-126)$ and decreased the activation of p38 MAPK (Fig. 4C and E).

Collectively, these results support the hypothesis that bvPLA 2 acts by increasing p-AKT protein levels and decreasing p-p38 protein levels to reduce $\operatorname{PrP}(106-126)$-mediated neurotoxicity.

\section{Discussion}

The purpose of this study was to explore a potential mechanism responsible for group III sPLA2-mediated protective effects against prion disease. The results support the efficacy of group III sPLA2, including bvPLA2 from Apis mellifica, for use in therapeutic strategies against prion-mediated neurodegeneration.

Prion diseases are a family of neurodegenerative disease characterized by neuronal vacuolation, gliosis, and astrocytosis triggered by conversion of PrPC to the scrapie-associated misfolded PrP isoform, PrPSc (1). PrP(106-126) possess physicochemical properties similar to PrPSc, including neurotoxicity and $\beta$-sheet formation (28-31). Some reports showed that $\operatorname{PrP}(106-126)$-mediated neurotoxicity is related to endoplasmic reticulum stress and mitochondrial disruption, and thereby to induction of AKT and p38 MAPK-dependent apoptosis $(29,32,33)$. In this study, $\operatorname{PrP}(106-126)$ induced apoptosis, inactivated PI3K/AKT pathways, and activated the p38 MAPK and caspase-3 pathways (Figs. 1 and 2). Interestingly, these neurotoxic pathways were inhibited by bvPLA2 treatment, resulting in a neuroprotective effect against $\operatorname{PrP}(106-126)$-mediated neuronal cell death (Figs. 1 and 2).

Some reports have shown that the activity of PLA2 influences the progression of neurodegenerative diseases including prion disease (34). Findings from the studies by Bate et al showed that inhibition of PLA2 inhibited prion-mediated neuronal cell death (35); however, this effect can differ depending on the PLA2 member (20). Several reports investigated how cPLA2 influences prion-mediated neurotoxicity while sPLA2 does not (34). Also, a recent study suggested that group III PLA2 prevents Alzheimer's disease in a transgenic mouse model (27). Consistent with this, bvPLA2-treated SH-SY5Y cells were protected against PrP(106-126)-mediated neuronal cell death.

Group III sPLA2, is a subfamily of secreted PLA2. A recent study suggested a therapeutic strategy to regulate Alzheimer's disease by enhancing $\alpha$-secretase-dependent APP processing $(19,27)$. As a member of group III sPLA2, bvPLA2 possesses structural and biochemical properties homologous to human group III PLA2, including the ability to regulate neurite outgrowth and mediate atherosclerosis $(26,36)$. A recent study showed that group III sPLA2, including bvPLA2, acts through modulation of PI3K/AKT signaling in neuritogenesis (19). Consistent with these results, $\operatorname{PrP}(106-126)$-induced AKT inactivation was regulated by bvPLA2 treatment in SH-SY5Y neuronal cells (Fig. 3E). These results indicate that bvPLA2mediated regulation of PI3K/AKT signaling may be a crucial target for the blockade of $\operatorname{PrP}(106-126)$-mediated neurotoxicity.

Some reports have shown that bee venom attenuates neuroinflammatory events by regulation of p38 MAPK signaling in neurodegenerative diseases $(36,37)$. However, the effect of bvPLA2 enzymes on p38 MAPK activity in neurodegenerative disorders has not been reported. In the present study, we showed that bvPLA 2 prevents $\operatorname{PrP}(106-126)$-mediated neuronal cell death by inactivation of p38 MAPK (Fig. 4).

It remains questionable whether gene regulation of human group III sPLA2 would produce similar effects to that by bvPLA2 administration to $\operatorname{PrP}(106-126)$-treated cells. Further study is required to illustrate the influence of human group III SPLA2 on PrP(106-126)-mediated neurotoxicity in vitro and/or in vivo. Collectively, the results suggest that bvPLA2 prevents neurotoxicity induced by $\operatorname{PrP}(106-126)$ through regulation of p38 MAPK and AKT activation. This is the first study to identify the neuroprotective effects of group III sPLA2 against PrP(106-126)-mediated neurotoxicity and also, suggests that prion-related neurodegenerative diseases may be attenuated by using group III sPLA2 activators, including bvPLA2 .

\section{Acknowledgements}

This study was supported by a grant from the Regional Research Universities Program and the Center for Healthcare Technology Development and the National Research Foundation of Korea funded by the Korean Government (2010-21492).

\section{References}

1. Brandner S, Klein MA, Frigg R, et al: Neuroinvasion of prions: insights from mouse models. Exp Physiol 85: 705-712, 2000.

2. Kretzschmar HA: Molecular pathogenesis of prion diseases. Eur Arch Psychiatry Clin Neurosci 249 (Suppl 3): S56-S63, 1999.

3. Avrahami D and Gabizon R: Age-related alterations affect the susceptibility of mice to prion infection. Neurobiol Aging (In press).

4. Diez M, Groth D, DeArmond SJ, Prusiner SB and Hokfelt T: Changes in neuropeptide expression in mice infected with prions. Neurobiol Aging 28: 748-765, 2007.

5. Jeong JK, Moon MH, Seol JW, Seo JS, Lee YJ and Park SY: Prion peptide-mediated cellular prion protein overexpression and neuronal cell death can be blocked by aspirin treatment. Int J Mol Med 27: 689-693, 2011.

6. Mallucci G, Dickinson A, Linehan J, Klohn PC, Brandner S and Collinge J: Depleting neuronal PrP in prion infection prevents disease and reverses spongiosis. Science 302: 871-874, 2003.

7. Radford HE and Mallucci GR: The role of GPI-anchored PrP C in mediating the neurotoxic effect of scrapie prions in neurons. Curr Issues Mol Biol 12: 119-127, 2010.

8. Sim VL and Caughey B: Ultrastructures and strain comparison of under-glycosylated scrapie prion fibrils. Neurobiol Aging 30: 2031-2042, 2009.

9. Didonna A and Legname G: Aberrant ERK 1/2 complex activation and localization in scrapie-infected GT1-1 cells. Mol Neurodegener 5: $29,2010$.

10. Freixes M, Rodriguez A, Dalfo E and Ferrer I: Oxidation, glycoxidation, lipoxidation, nitration, and responses to oxidative stress in the cerebral cortex in Creutzfeldt-Jakob disease. Neurobiol Aging 27: 1807-1815, 2006. 
11. Seo JS, Seol JW, Moon MH, Jeong JK, Lee YJ and Park SY: Hypoxia protects neuronal cells from human prion protein fragment-induced apoptosis. J Neurochem 112: 715-722, 2010.

12. Jeong JK, Seol JW, Moon MH, et al: Cellular cholesterol enrichment prevents prion peptide-induced neuron cell damages Biochem Biophys Res Commun 401: 516-520, 2010.

13. Seo JS, Moon MH, Jeong JK, et al: SIRT1, a histone deacetylase, regulates prion protein-induced neuronal cell death. Neurobio Aging: Nov 11, 2010 (Epub ahead of print).

14. Gu Y, Fujioka H, Mishra RS, Li R and Singh N: Prion peptide 106-126 modulates the aggregation of cellular prion protein and induces the synthesis of potentially neurotoxic transmembrane PrP. J Biol Chem 277: 2275-2286, 2002.

15. Forloni G, Angeretti N, Chiesa R, et al: Neurotoxicity of a prion protein fragment. Nature 362: 543-546, 1993.

16. Singh N, Gu Y, Bose S, Kalepu S, Mishra RS and Verghese S: Prion peptide 106-126 as a model for prion replication and neurotoxicity. Front Biosci 7: a60-a71, 2002.

17. Mingarro I, Perez-Paya E, Pinilla C, Appel JR, Houghten RA and Blondelle SE: Activation of bee venom phospholipase A2 through a peptide-enzyme complex. FEBS Lett 372: 131-134, 1995.

18. Rodriguez De Turco EB, Jackson FR, DeCoster MA, Kolko M and Bazan NG: Glutamate signalling and secretory phospholipase A2 modulate the release of arachidonic acid from neuronal membranes. J Neurosci Res 68: 558-567, 2002.

19. Masuda S, Yamamoto K, Hirabayashi T, et al: Human group III secreted phospholipase A2 promotes neuronal outgrowth and survival. Biochem J 409: 429-438, 2008.

20. Kudo I and Murakami M: Phospholipase A2 enzymes. Prostaglandins Other Lipid Mediat 68-69: 3-58, 2002.

21. Scott DL, Otwinowski Z, Gelb MH and Sigler PB: Crystal structure of bee-venom phospholipase A2 in a complex with a transition-state analogue. Science 250: 1563-1566, 1990.

22. Burke JE and Dennis EA: Phospholipase A2 structure/function, mechanism, and signaling. J Lipid Res 50 (Suppl): S237-S242, 2009.

23. Sun GY, Shelat PB, Jensen MB, He Y, Sun AY and Simonyi A: Phospholipases A2 and inflammatory responses in the central nervous system. Neuromolecular Med 12: 133-148, 2010.

24. Sato H, Kato R, Isogai Y, et al: Analyses of group III secreted phospholipase A2 transgenic mice reveal potential participation of this enzyme in plasma lipoprotein modification, macrophage foam cell formation, and atherosclerosis. J Biol Chem 283: 33483-33497, 2008.

25. Sato H, Taketomi Y, Isogai Y, et al: Group III secreted phospholipase A2 regulates epididymal sperm maturation and fertility in mice. J Clin Invest 120: 1400-1414, 2010.
26. Valentin E, Ghomashchi F, Gelb MH, Lazdunski $M$ and Lambeau G: Novel human secreted phospholipase A(2) with homology to the group III bee venom enzyme. J Biol Chem 275: 7492-7496, 2000

27. Yang X, Sheng W, He Y, et al: Secretory phospholipase A2 type III enhances alpha-secretase-dependent amyloid precursor protein processing through alterations in membrane fluidity. J Lipid Res 51: 957-966, 2010.

28. Melo JB, Agostinho P and Oliveira CR: Prion protein aggregation and neurotoxicity in cortical neurons. Ann NY Acad Sci 1096: 220-229, 2007.

29. Corsaro A, Thellung S, Villa V, et al: Prion protein fragment 106-126 induces a p38 MAP kinase-dependent apoptosis in SH-SY5Y neuroblastoma cells independently from the amyloid fibril formation. Ann NY Acad Sci 1010: 610-622, 2003.

30. O'Donovan CN, Tobin D and Cotter TG: Prion protein fragment PrP-(106-126) induces apoptosis via mitochondrial disruption in human neuronal SH-SY5Y cells. J Biol Chem 276: 43516-43523, 2001.

31. Sakudo A and Ikuta K: Prion protein functions and dysfunction in prion diseases. Curr Med Chem 16: 380-389, 2009.

32. Schneider B, Mutel V, Pietri M, Ermonval M, Mouillet-Richard S and Kellermann O: NADPH oxidase and extracellular regulated kinases $1 / 2$ are targets of prion protein signaling in neuronal and nonneuronal cells. Proc Natl Acad Sci USA 100: 13326-13331, 2003.

33. Pietri M, Caprini A, Mouillet-Richard S, et al: Overstimulation of PrPC signaling pathways by prion peptide 106-126 causes oxidative injury of bioaminergic neuronal cells. J Biol Chem 281: 28470-28479, 2006.

34. Bate C, Salmona M, Diomede L and Williams A: Squalestatin cures prion-infected neurons and protects against prion neurotoxicity. J Biol Chem 279: 14983-14990, 2004.

35. Bate C, Tayebi M, Diomede L, Salmona M and Williams A: Glimepiride reduces the expression of PrPc, prevents PrPSc formation and protects against prion mediated neurotoxicity in cell lines. PLoS One 4: e8221, 2009.

36. Lee WR, Kim SJ, Park JH, et al: Bee venom reduces atherosclerotic lesion formation via anti-inflammatory mechanism. Am J Chin Med 38: 1077-1092, 2010.

37. Yang EJ, Jiang JH, Lee SM, et al: Bee venom attenuates neuroinflammatory events and extends survival in amyotrophic lateral sclerosis models. J Neuroinflammation 7: 69, 2010. 